\title{
Investigating the Association of Relative Central Blood Pressure Indices and Severity of Coronary Artery Disease
}

\author{
Pouria Ali Forouzanmehr (iD ${ }^{1,}{ }^{,}$, Ebrahim Haidary Sardaby ${ }^{2}$ and Saeed Hesam ${ }^{3}$ \\ ${ }^{1}$ Department of Cardiovascular Diseases, Ahvaz Jundishapur University of Medical Sciences, Ahvaz, Iran \\ ${ }^{2}$ Department of Cardiovascular Diseases, Atherosclerosis Research Center, Ahvaz Jundishapur University of Medical Sciences, Ahvaz, Iran \\ ${ }^{3}$ Department of Biostatistics and Epidemiology, Faculty of Public Health, Ahvaz Jundishapur University of Medical Sciences, Ahvaz, Iran \\ "Corresponding author: Department of Cardiovascular Diseases, Ahvaz Jundishapur University of Medical Sciences, Ahvaz, Iran. Tel: +98-6132228037, Fax: +98-6132216104, Email: \\ forouzanmehr.p@ajums.ac.ir
}

Received 2019 August 11; Revised 2019 September 15; Accepted 2019 September 21

\begin{abstract}
Background: Central blood pressure is an important index in central hemodynamic stress and may be associated with an increased risk of cardiovascular diseases.

Objectives: This study evaluated the central blood pressure indices in coronary artery disease (CAD) subjects and their association with the number of diseased vessels involved in CAD.

Methods: A random sample of 300 Iranian subjects who underwent coronary angiography (mean age $58.08 \pm 10.62$ years) was studied. Coronary lesions were defined as luminal stenosis $\geq 50 \%$ and the number of diseased vessels and the central aortic pressure were taken. The probability of central blood pressure indices with the number of diseased vessels was also determined by using cutoff points, based on the sensitivity, specificity, positive predictive value, and negative predictive value.

Results: The central blood pressure indices (fractional pulse pressure (FPP), systolic pressure (FSP), diastolic pressure (FDP) and FSP/FDP) were statistically significant in CAD patients with an increase in the number of diseased vessels $(\mathrm{P}<0.001)$. There was a significant association between central blood pressure indices and $\mathrm{CAD}(\mathrm{P}<0.001)$ as well as with the increase in the number of diseased vessels $(\mathrm{P}<0.001)$. Both odds ratio (11.84; 95\% CI: 6.11-22.95; $\mathrm{P}<0.001$ ) and ROC analysis (AUC: 0.909; $95 \%$ CI: 0.870 - 0.939; $\mathrm{P}$ $<0.001)$ showed FSP as the strongest predictor of CAD. Furthermore, cut-off points of the $\operatorname{FSP}(\geq 1.373), \operatorname{FDP}(\leq 0.811), \operatorname{FPP}(\geq 0.559)$ and FSP/FDP ( $\geq 1.688$ ) were determined for CAD for the first time based on $92 \%$ sensitivity, and $74 \%$ specificity.

Conclusions: FDP, FSP, and FSP, in particular, could be the effective predictors of CAD in Iranian populations. Therefore, the quality of life may be improved by the management of central blood pressure and using CAD cut-off points.
\end{abstract}

Keywords: Coronary Angiography, Blood Pressure, Coronary Artery Disease, Aortic Pressure

\section{Background}

Coronary artery disease (CAD) is one of the major causes of mortality and morbidity in industrial societies, for which there are many risk factors (1). Age, sex, smoking, dyslipidemia, diabetes mellitus and hypertension are associated with the severity of coronary lesions in angiography (2). Increased brachial blood pressure is one of the most important risk factors for cardiovascular events in the future. However, the brachial blood pressure does not accurately reflect central aortic blood pressure, as it may indicate a false increase in isolated peripheral systolic blood pressure despite normal aortic blood pressure (3). However, some studies have demonstrated that more than $30 \%$ of men and $10 \%$ of women with normal brachial blood pressure had central blood pressure similar to individuals with stage 1 hypertension (4). New evidence suggests the central blood pressure may predict cardiovascular events and end-organ damage associated with hypertension better than the brachial pressure (5-7).

Central blood pressure is a useful index for central hemodynamic stress and may be associated with an increased risk of cardiovascular diseases. Moreover, central blood pressure is suggested as a more reliable marker for cardiovascular events rather than the brachial pressure measurement $(3,8)$. However, the relationship between central blood pressure parameters and the severity of coronary artery disease has not been well addressed $(3,9)$. Central aortic pressure indices such as pulse pressure (PP), fractional systolic pressure (FSP) and fractional diastolic pressure (FDP) may be associated with an increased risk of cardiovascular disease. Additionally, according to studies, the severity of coronary artery disease can be predicted by 
measuring FSP and FDP (10). Furthermore, a higher central $\mathrm{PP}$ is associated with extensive CAD in angiography (11).

\section{Objectives}

This study aimed to assess the relationship between the severity of coronary artery disease and central aortic blood pressure. Moreover, the probability of CAD and 3VD has been determined for the first time by using cut-off points based on sensitivity, specificity, NPV, and PPV in an Iranian population.

\section{Methods}

\subsection{Study Population}

This study was a hospital-based cross-sectional study conducted at the Center for Elective Diagnostic Coronary Angiography, Ahvaz, Iran. The study population included a total of 300 subjects within an age range of $<18$ years old, who were selected, based on the simple random sampling method and the following selection criteria from December 2017 to December 2018.

\subsection{Selection Criteria}

Patients with the following conditions were excluded from this study: no sinus rhythm, aortic valve stenosis or regurgitation more than mild severity and other valves more than moderate severity, renal impairment with 1.5 $<$ serum creatinine, peripheral artery disease, CABG cases, and contraindications of coronary artery angiography. Moreover, patients who had MI or thromboembolism in the last three months were excluded from the study to prevent any interference with the analysis results. Informed written consent was obtained from all subjects. The study was approved by the ethical review board at Ahvaz University of Medical Sciences (ethical ID number: IR.AJUMS.REC.1397.016).

\subsection{Data Collection and Central Blood Pressure Measurements}

The main risk factors of CAD were extracted for all patients, based on history, examination, and medical record data. After which they underwent angiography. The central aortic blood pressure of the patients was measured during angiography. An angiographic catheter was placed in an ascending aorta and connected to a pressure transducer with a fluid-filled system to measure central systolic blood pressure (SBP) and central diastolic blood pressure (DBP).

Mean arterial pressure (MAP) and arterial pulse pressure (PP) were obtained to calculate FSP, FDP and FPP, which were $\mathrm{FSP}=\mathrm{SBP} / \mathrm{MAP}$, FDP $=\mathrm{DBP} / \mathrm{MAP}, \mathrm{FPP}=\mathrm{PP} / \mathrm{MAP}$, respectively.

\subsection{Determine the Number of Diseased Vessels}

The severity of coronary artery disease was also calculated by the cardiologist, and the results were divided into two groups: NO CAD and CAD. The CAD cases were then classified based on the severity of CAD with more than $50 \%$ stenosis in the form of one vessel disease (1VD), two-vessel disease (2VD), and three-vessel disease (3VD).

\subsection{Statistical Analysis}

In order to determine the significance of different parameters, the data collected during the current study were recorded and statistically analyzed by the SPSS program (version 22.0) and Medcalc (version 15.0). Results were expressed as mean \pm SD. The Kolmogorov-Smirnov test and Q-Q plot were used to study the normality of the data.

Univariate data analysis was conducted using an independent $t$-test, ANOVA test, and Spearman correlation coefficient. Multivariate data analysis was carried out using ordinal regression and logistic regression. Moreover, the ROC curve was used to assess the diagnostic power of variables, and the appropriate cut-off points were obtained based on sensitivity, specificity, positive predictive value, and negative predictive value. The significance level was considered to be 0.05 .

\section{Results}

Out of 300 subjects, the coronary artery angiography of whom was recorded, 211 patients (59.6 years \pm 9.6) were classified as CAD and 89 individuals ( 54.45 years \pm 11.9 ) as NO CAD. There were 163 (54.3\%) males and 137 (45.7\%) females (data not shown). The mean relative indices of central blood pressure (FPP, FSP, FDP, and FSP/FDP) in NO CAD patients and patients with $1 \mathrm{VD}, 2 \mathrm{VD}$, and $3 \mathrm{VD}$ are presented in Table 1 . There were significant differences in all central blood pressure indices in these groups $(\mathrm{P}<0.001)$.

Odds ratio (OR) for the FPP, FSP, FDP, and FSP/FDP was found to be significant $(\mathrm{P}<0.001)$ in $\mathrm{CAD}$ and $3 \mathrm{VD}$ subjects (Table 2). According to ordinal regression analysis in which variables such as age and sex were matched, there was a relationship between the relative indices of central blood pressure and the number of diseased vessels such that the higher the number of diseased vessels, the higher the FPP, FSP, FSP/FDP and the lower the FDP $(\mathrm{P}<0.001)$ (Table 3$)$.

The ROC curve was used to evaluate the predictive power of central blood pressure indices (Table 4). The ROC curve for SBP, DBP, FPP, FSP, FDP and FSP/FDP was 0.672, 0.757, 0.909, 0.909, 0.909, and 0.909 for CAD diagnosis, respectively. All central blood pressure indices (especially relative indices) had predictive power for CAD diagnosis ( $\mathrm{P}<$ 


\begin{tabular}{lccccc}
\hline \multicolumn{1}{l}{ Table 1. Comparisons of the Relative Indices of Central Blood Pressure in NO CAD Patients and the Number of Coronary Artery Disease (1VD, 2VD, 3VD) } & & 2 \\
\hline & NO CAD & CAD (1VD, 2VD, 3VD) & 1VD & 2VD & PVD \\
\hline FPP & $0.52 \pm 0.10$ & $0.76 \pm 0.14$ & $0.62 \pm 0.11$ & $0.74 \pm 0.10$ & $0.88 \pm 0.09$ \\
FSP & $1.34 \pm 0.07$ & $1.50 \pm 0.09$ & $1.41 \pm 0.07$ & $1.49 \pm 0.06$ & 0.001 \\
FDP & $0.82 \pm 0.03$ & $0.74 \pm 0.04$ & $0.79 \pm 0.03$ & $0.75 \pm 0.03$ & $0.70 \pm 0.03$ \\
FSP/FDP & $1.63 \pm 0.16$ & $2.04 \pm 0.27$ & $1.80 \pm 0.19$ & $2.00 \pm 0.19$ & $2.26 \pm 0.19$ \\
\hline
\end{tabular}

Abbreviations: CAD, coronary artery disease; FDP, fractional diastolic pressure; FPP, fractional pulse pressure; FSP, fractional systolic pressure; 1VD, one vessel disease; $2 \mathrm{VD}$, two vessel disease; $3 \mathrm{VD}$, three vessel disease

${ }^{a}$ P Value: comparison between NO CAD and CAD

Table 2. Odds Ratio According to the Relative Indices of Central Blood Pressure for the Diagnosis of CAD (vs. NO CAD Patients) and the Occurrence of 3VD (vs. the Tota Patients with 1VD and 2VD)

\begin{tabular}{|cccc|}
\hline & & OR $(\% 95$ CI $)$ & P Value \\
\hline CAD & & $2.76(2.09,3.64)$ & $<0.001$ \\
& FSP/SDP & $5.20(3.34,8.08)$ & $<0.001$ \\
& FPP & $11.84(6.11,22.95)$ & $<0.001$ \\
& FSP & $0.007(0.002,0.03)$ & $<0.001$ \\
\hline & FDP & & $<0.001$ \\
\hline 3VD & & $2.28(1.82,2.85)$ & $<0.001$ \\
& FSP/SDP & $4.85(3.15,7.47)$ & $<0.001$ \\
& FPP & $10.68(5.59,20.43)$ & $<0.001$ \\
\hline & FSP & $0.009(0.002,0.032)$ & \\
\hline & FDP & & \\
\hline
\end{tabular}

Abbreviations: CAD, coronary artery disease; $\mathrm{CI}$, confidence interval; FDP, fractional diastolic pressure; FPP, fractional pulse pressure; FSP, fractional systolic pressure; OR, odds ratio; 1VD, one vessel disease; 2VD, two vessel disease; 3VD, three vessel disease

Table 3. Association of Relative Indices of Central Blood Pressure and the Number of Coronary Arteries Disease $(0=$ No CAD, $1=1 \mathrm{VD}, 2=2 \mathrm{VD}, 3=3 \mathrm{VD})$ Matched for Age and Gender by Linear Regression Models

\begin{tabular}{lccc}
\hline & $\beta$ & \%95 CI & PValue \\
\hline FSP/FDP & 9.227 & $(7.792,10.662)$ & $<0.001$ \\
FPP & 16.22 & $(13.73,18.71)$ & $<0.001$ \\
FSP & 24.32 & $(20.59,28.06)$ & $<0.001$ \\
FDP & -48.65 & $(-56.12,-41.18)$ & $<0.001$ \\
\hline
\end{tabular}

Abbreviations: $\beta$, ordinal regression coefficient; $\mathrm{CAD}$, coronary artery disease; $\mathrm{CI}$, confidence interval; FDP, fractional diastolic pressure; FPP, fractional pulse pressure; FSP, fractional systolic pressure; 1VD, one vessel disease; $2 \mathrm{VD}$, two vessel disease; 3VD, three vessel disease

0.001). Moreover, the ROC curve for central blood pressure indices and the predictive power of the main 3VD was $0.679,0.676,0.894,0.894,0.894$, and 0.894 , respectively. Thus, it can be concluded that central blood pressure indices have predictive power for an increase in the number of diseased vessels $(\mathrm{P}<0.001)$. This diagnostic power of $3 \mathrm{VD}$ in $\mathrm{CAD}$ patients for relative indices of central blood
Table 4. The AUC of the Relative Indices of Central Blood Pressure for the Diagnosis of CAD (vs. NO CAD Patients) and the Occurrence of 3VD (vs. the Total Patients with IVD and 2VD) Using the ROC Curve

\begin{tabular}{|c|c|c|c|}
\hline & AUC & \%95 CI & PValue \\
\hline \multicolumn{4}{|l|}{ CAD } \\
\hline SBP & 0.672 & $(0.616,0725)$ & $<0.001$ \\
\hline DBP & 0.757 & $(0.704,0.804)$ & $<0.001$ \\
\hline FSP/FDP & 0.909 & $(0.870,0.939)$ & $<0.001$ \\
\hline FPP & 0.909 & $(0.870,0.939)$ & $<0.001$ \\
\hline FSP & 0.909 & $(0.870,0.939)$ & $<0.001$ \\
\hline FDP & 0.909 & $(0.870,0.939)$ & $<0.001$ \\
\hline \multicolumn{4}{|l|}{ 3VD } \\
\hline SBP & 0.679 & $(0.611,0.741)$ & $<0.001$ \\
\hline DBP & 0.676 & $(0.609,0.739)$ & $<0.001$ \\
\hline FSP/FDP & 0.894 & $(0.845,0.932)$ & $<0.001$ \\
\hline FPP & 0.894 & $(0.845,0.932)$ & $<0.001$ \\
\hline FSP & 0.894 & $(0.845,0.932)$ & $<0.001$ \\
\hline FDP & 0.894 & $(0.845,0.932)$ & $<0.001$ \\
\hline
\end{tabular}

Abbreviations: AUC, area of ROC curve; CAD, coronary artery disease; CI, confidence interval; FDP, fractional diastolic pressure; FPP, fractional pulse pressure; FSP, fractional systolic pressure; 1VD, one vessel disease; $2 \mathrm{VD}$, two vessel disease; 3VD, three vessel disease

pressure (FPP, FDP, FSP, FSP/FDP) is higher than that of absolute indices (SBP, DBP).

Regarding the significant relationship between central blood pressure indices, CAD and 3VD, two cut-off points were determined; once with high sensitivity and the next time with high specificity, positive predictive value (PPV) and negative predictive value (NPV) at both cut-off points (Table 5). For example, the cut-off point of the FSP index for CAD group (all patients with 1VD, 2VD, and 3VD) was $\leq 1.373$ based on $92 \%$ sensitivity, and $74 \%$ specificity. Another cutoff point of the same index was $\leq 1.421$ with a sensitivity of about $77 \%$, but a specificity of about $93 \%$. 


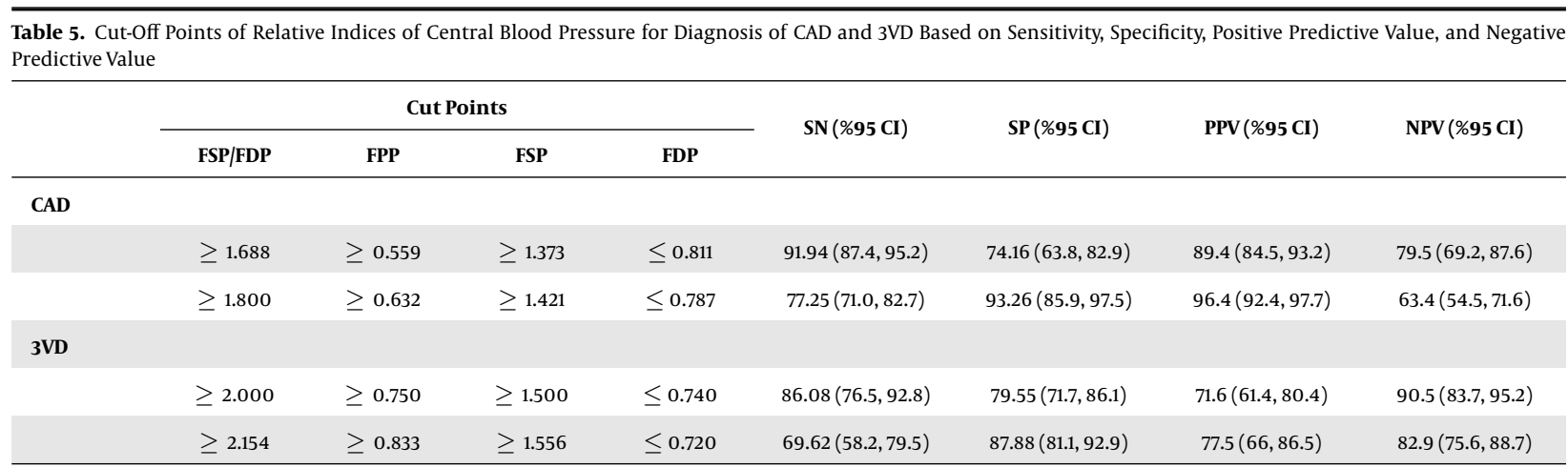

Abbreviations: CAD, coronary artery disease; CI, confidence interval; FDP, fractional diastolic pressure; FPP, fractional pulse pressure; FSP, fractional systolic pressure; $\mathrm{NPV}$, negative predictive value; PPV, positive predictive value; SN, sensitivity; SP, specificity; 3VD, three vessel disease

\section{Discussion}

Studies have been conducted on central aortic blood pressure indices such as FPP, FSP and FDP, and their association with cardiovascular outcomes, confirming the association of these indices with the increased risk of cardiovascular disease and an increase in the severity of coronary artery disease $(10,12,13)$. Previous studies have reported the association between central blood pressure and cardiovascular diseases $(3,5,6)$. Moreover, Jankowski et al. showed that PP, FSP, and FDP of the central aorta were associated with the risk of $3 \mathrm{VD}$ in CAD patients. They stated that the risk of $3 \mathrm{VD}$ increases by $15 \%, 28 \%$, and $39 \%$ per $10 \mathrm{mmHg}$ increases in the central PP, per 0.1 increases in FSP and per 0.1 reductions in FDP, respectively (14). As observed in this study, there was a significant association between increased FPP, FSP and FSP/FDP, decreased FDP, and the higher risk of 3VD (FPP 4.85, FSP 10.68, FDP 0.009 and FSP/FDP 2.28) $(\mathrm{P}<0.001)$. Moreover, there was a significant correlation between increases in central blood pressure indices (FSP/FDP, FSP, FPP), reduction of FDP as well as increases in the number of diseased vessels. However, both odds ratio (11.84; 95\% CI: 6.11 - 22.95; P < 0.001) and ROC analysis (AUC: 0.909; 95\% CI: 0.870 - 0.939; P < 0.001) showed FSP as the strongest predictor of CAD. In another study, Nakayama et al. showed that FDP, but not FSP, is independently associated with CAD risk in patients undergoing coronary angiography (11). In our study, it was shown that lower FDP values, as well as higher FSP values, are associated with an increased risk of CAD. It means that a lower level of FDP accompanied by increases in other central blood pressure indices could be a suitable marker for predicting the number of involved vessels.

Moreover, it was shown that there is an association between the central blood pressure indices, in particular, its relative indices (FPP, FDP, FSP, and FSP/FDP), in patients undergoing $\mathrm{CAD}$ and an increase in the number of diseased vessels $(\mathrm{P}<0.001)$. Furthermore, The ROC curve suggested that FPP, FDP, FSP, FSP/FDP have higher predictive power than absolute indices (SBP and DBP) for the occurrence of $\mathrm{CAD}$ and also an increase in the number of diseased vessels $(\mathrm{P}<0.001)$ (Table 4).

In this study, the probability of CAD and 3VD was predicted for the first time by using cut-off points based on sensitivity, specificity, NPV, and PPV. The cut-off points of the FSP $(\geq 1.373), \operatorname{FDP}(\leq 0.811), \mathrm{FPP}(\geq 0.559)$ and FSP/FDP ( $\geq 1.688$ ) for CAD were determined in this study based on 92\% sensitivity and 74\% specificity. Additionally, cut-off points of $\operatorname{FSP}(\geq 1.500), \operatorname{FDP}(\leq 0.740), \operatorname{FPP}(\geq 0.750)$ and FSP/FDP $(\geq 2.000)$ were determined for 3VD with $86 \%$ sensitivity and $80 \%$ specificity.

\subsection{Conclusions}

Central blood pressure indices, such as FPP, FDP, FSP, FSP/FDP may be associated with increased diseased vessels in CAD. However, FSP has been shown as the strongest predictor of CAD in Iranian populations. Therefore, the quality of life may be improved by the management of blood pressure treatment based on central blood pressure control and using cut-off points.

\subsection{Study Limitations}

1. There were a limited number of patients, and we used a small group who were referred for elective CAG.

2. A fluid-filled system was used to record coronary BP, while the use of a high-fidelity pressure transducer can increase the accuracy of the recorded pressure in this study. So, high-fidelity pressure transducer might be used as an advanced pressure measurement device in future clinical studies. 


\section{Acknowledgments}

We would like to thank the financial support of Deputy for Research of the Ahvaz Jundishapur University of Medical Sciences.

\section{Footnotes}

Authors' Contribution: Study concept and design: Pouria Ali Forouzanmehr and Ebrahim Haidary Sardaby. Analysis and interpretation of data: Pouria Ali Forouzanmehr. Drafting of the manuscript: Pouria Ali Forouzanmehr. Critical revision of the manuscript: Pouria Ali Forouzanmehr, Ebrahim Haidary Sardaby, and Saeed Hesam. Statistical analysis: Saeed Hesam.

Conflict of Interests: We wish to confirm that there are no known conflicts of interest associated with this publication and there has been no significant financial support for this work that could have influenced its outcome.

Ethical Approval: The study was approved by the ethical review board at Ahvaz University of Medical Sciences (ethical ID number: IR.AJUMS.REC.1397.016).

Funding/Support: This research received no specific grant from any funding agency in the public, commercial, or not-for-profit sectors.

Patient Consent: Informed written consent was obtained from all subjects.

\section{References}

1. Suwaidi JA, Hamasaki S, Higano ST, Nishimura RA, Holmes DR, Lerman A. Long-term follow-up of patients with mild coronary artery disease and endothelial dysfunction. Circulation. 2000;101(9):948-54. doi: 10.1161/01.cir.101.9.948. [PubMed: 10704159].

2. Mohagheghi A, Mohebi M, Hedayat DK, Tabatabaee A, Naseri N. The relationship of Gensini score with the cardiovascular risk of patients with indication of angiography. Tehran Univ Med J. 2011;69(6):388-91.

3. Nelson MR, Stepanek J, Cevette M, Covalciuc M, Hurst RT, Tajik AJ. Noninvasive measurement of central vascular pressures with arterial tonometry: Clinical revival of the pulse pressure waveform? Mayo
Clin Proc. 2010;85(5):460-72. doi: 10.4065/mcp.2009.0336. [PubMed: 20435839]. [PubMed Central: PMC2861976].

4. Avolio A. Central aortic blood pressure and cardiovascular risk: A paradigm shift? Hypertension. 2008;51(6):1470-1. doi: 10.1161/HYPERTENSIONAHA.107.108910. [PubMed: 18426994].

5. McEniery CM, Cockcroft JR, Roman MJ, Franklin SS, Wilkinson IB. Central blood pressure: Current evidence and clinical importance. Eur Heart J. 2014;35(26):1719-25. doi: 10.1093/eurheartj/eht565. [PubMed: 24459197]. [PubMed Central: PMC4155427].

6. Patel BC, Gupta R, Latif F. Indices of central aortic blood pressure and their impact on cardiovascular outcomes. J Cardiovasc Med (Hagerstown). 2012;13(1):38-45. doi: 10.2459/JCM.ob013e32834ead20. [PubMed: 22130043].

7. Townsend RR, Roman MJ, Najjar SS, Cockcroft JR, Feig PU, Stockbridge NL. Central blood pressure measurements-an opportunity for efficacy and safety in drug development? J Am Soc Hypertens. 2010;4(5):211-4. doi: 10.1016/j.jash.2010.07.002. [PubMed: 20729159].

8. Pini R, Cavallini MC, Palmieri V, Marchionni N, Di Bari M, Devereux RB, et al. Central but not brachial blood pressure predicts cardiovascular events in an unselected geriatric population: The ICARe Dicomano study. J Am Coll Cardiol. 2008;51(25):2432-9. doi: 10.1016/j.jacc.2008.03.031. [PubMed: 18565402].

9. Sharma KH, Sharma N, Shah K, Patil S. Impact of coronary artery disease on augmentation index as measured by estimated central blood pressure: A case control study in Asian Indians. Indian Heart J. 2018;70(5):615-21. doi: 10.1016/j.ihj.2017.12.001. [PubMed: 30392497]. [PubMed Central: PMC6372218].

10. Song-Tao A, Yan-Yan Q, Li-Xia W. The severity of coronary artery disease evaluated by central systolic pressure and fractional diastolic pressure. N Am J Med Sci. 2010;2(5):218-20. doi: 10.4297/najms.2010.2218. [PubMed: 22574292]. [PubMed Central: PMC3347647].

11. Khoueiry G, Azab B, Torbey E, Abi Rafeh N, Atallah JP, Ahern K, et al. Aortic pulse pressure is associated with the localization of coronary artery disease based on coronary flow lateralization. Am J Hypertens. 2012;25(10):1055-63. doi: 10.1038/ajh.2012.87. [PubMed: 22739807]. [PubMed Central: PMC3733062].

12. Nakayama Y, Hayashi T, Yoshimaru K, Tsumura K, Ueda H. Low fractional diastolic pressure in the ascending aorta increased the risk of coronary heart disease. J Hum Hypertens. 2002;16(12):837-41. doi: 10.1038/sj.jhh.1001489. [PubMed: 12522464].

13. Nanduri S, Tuwairqi K, Velagapudi K, Saidi A, Pinzon OW, Dranow E, et al. Central Fractional Pulse Pressure Predicts Cardiac Power Index in Patients with Acute Coronary Syndrome. J Am Coll Cardiol. 2017;69(11):1197. doi: 10.1016/s0735-1097(17)34586-2.

14. Jankowski P, Kawecka-Jaszcz K, Bryniarski L, Czarnecka D, Brzozowska-Kiszka M, Posnik-Urbanska A, et al. Fractional diastolic and systolic pressure in the ascending aorta are related to the extent of coronary artery disease. Am J Hypertens. 2004;17(8):641-6. doi: 10.1016/j.amjhyper.2004.01.009. [PubMed: 15288880]. 